\title{
Method of calculating heat transfer in furnaces of small power
}

\author{
Pavel Khavanov ${ }^{1, *}$ \\ ${ }^{1}$ Moscow state university of civil engineering, Yaroslavskoye sh. 26, 129337 Moscow, Russia
}

\begin{abstract}
This publication presents the experiences and results of generalization criterion equation of importance in the analysis of the processes of heat transfer and thermal calculations of low-power heat generators cooled combustion chambers. With generalizing depending estimated contribution of radiation and convective heat transfer component in the complex for the combustion chambers of small capacity boilers. Determined qualitative and quantitative dependence of the integrated radiative-convective heat transfer from the main factors working combustion chambers of small volume.
\end{abstract}

Study of heat transfer in cooled combustion chambers and study the effect on the intensity of various factors is difficult and urgent task.

From the analysis of theoretical and experimental data on heat transfer in combustion chambers of various plants it should be that the existing methods do not take into account the peculiarities of heat transfer in furnaces of small dimensions and can't be used for their thermal design.

The process of heat transfer in furnaces from the radiating flow of combustion products is determined by the intensity of convection, molecular and radiative transfer, depending on the conditions of entry, ignition and combustion of the fuel-air mixture, changes in physicochemical properties of the medium, the hydrodynamic process conditions and other factors. Convective heat transfer, radiation and molecular thermal conductivity as heat transfer components of the complex, depending on the conditions of development of the process and make a different to-quantitative contribution to the resulting heat transfer. Neglecting any of these components without a detailed analysis of the process may lead to a distortion of the actual physical side of heat transfer and, consequently, to incorrect quantitative relations in the process. The same result is obtained and the analysis of the individual contribution of each component without consideration of their mutual influence [1].

Analysis of heat transfer by convection processes, molecular and turbulent heat conduction and radiation in the flow of gas leads to a system of differential equations to be solved simultaneously. The mathematical difficulties in solving this system, make it necessary to search for an approximate solution methods. The basic assumption in this case is connected with the exception of the integral members of the energy transfer equations, which are introduced into it as a result of the presence of radiation. The operation exception

\footnotetext{
${ }^{*}$ Corresponding author: roverton@mail.ru
} 
is easiest to implement in extreme cases, the optical thickness of the emitting layer: a very large and very small.

The intensity transfer by radiation heat in a combustion chamber of small volume and the furnaces of steam generators and process furnaces may be different even with the same temperature conditions as the optical properties of the layer emitting the combustion products in the cells of large and small volume differ in their numerical values in relation to those in the flue cells in a small volume of heat exchange formulation objectives of the study becomes important to change the range of the optical properties of the combustion environment, since it is known that the physical model of the process to determine the transfer of energy by radiation and the final results depend on the optical density of the medium to a large extent.

Calculated study the optical properties of the medium [2] in a small size furnace chambers showed that calculation methods regardless of the average absorption coefficient emitting medium is neither optically thin or optically thick.

For intermediate values of the optical thickness of the emitting layer to reveal a natural process requires additional experimental studies, because in some cases it is possible to use absorption coefficients averaged Planck (approximation of an optically thin layer) or the Rosseland (zoom optically thick layer) [3].

Analysis of experimental data has shown that in the investigated range of the optical thickness of a generalization of the experimental data obtained in the installation described below, may be made based on the absorption coefficients averaged Rosseland. Integral heat transfer is possible to determine the number of functional integral heat exchange:

$$
K_{\mathrm{T}}=\frac{I_{t h}-I_{e g}}{I_{t h}-I_{w}},
$$

$I_{t h}$ - enthalpy of combustion products at the theoretical combustion temperature, $\mathrm{kJ} / \mathrm{kg}$; $I_{e g}$ - enthalpy of combustion products at a temperature of exhaust gases, $\mathrm{kJ} / \mathrm{kg} ; I_{w}-$ enthalpy of combustion products at the wall, $\mathrm{kJ} / \mathrm{kg}$.

In this case, $K_{\mathrm{T}}$ determines the performance of the combustion chamber and a heat exchange function between the moving gas stream and radiating heating surface of the combustion chamber [4].

Heat transfer from the volume of the combustion chamber to its walls through the boundary layer due to radiation and turbulent diffusion (molecular transfer in this case can be neglected).

In the boundary layer and the heat transfer wall to the wall is carried out and the resulting thermal conductivity of molecular radiation medium to the heating surface. heat balance equation for the surface corresponding to the unit length of the cylindrical chamber, while using the diffusion approximation for energy transfer by radiation is written as follows:

$$
-D_{\text {turb }} \cdot \frac{d\left(\rho c_{p} T\right)}{d_{b}}-\lambda_{\text {rad }} \frac{d T}{d_{b}}=\frac{\sigma_{o} n^{2}\left(T_{c}^{4}-T_{w}^{4}\right)}{\frac{1}{\delta_{w}}-\frac{1}{2}}-\lambda_{m o l} \frac{d T}{d_{\Gamma}},
$$

$D_{\text {turb }}$ - turbulent diffusion coefficient, $\mathrm{m}^{2} / \mathrm{s} ; r$ - camera radius, $\mathrm{m} ; \mathrm{c}_{p}$ - isobaric heat capacity of the combustion products, $\mathrm{kJ} /(\mathrm{kg} \cdot \mathrm{K}) ; \rho$ - the density of the combustion products, $\mathrm{kg} / \mathrm{m}^{3}$; $\lambda_{\text {rad }}$ - radiative heat transfer coefficient of the Rosseland, $\mathrm{W} /(\mathrm{m} \cdot \mathrm{K}) ; \sigma_{\mathrm{o}}-$ Stefan-Boltzman constant, $\mathrm{W} /\left(\mathrm{m}^{2} \cdot \mathrm{K}^{4}\right) ; n-$ index of refraction; $\delta_{\mathrm{w}}-$ the emissivity of the surface; $\lambda_{\text {mol }}-$ molecular thermal conductivity, $\mathrm{W} /(\mathrm{m} \cdot \mathrm{K}) ; \mathrm{d}_{\mathrm{b}}$ - the diameter of the burner, $\mathrm{m}$. 
Equation (2) is written for surfaces that obey the law of Kirchgoff. From the resulting heat balance equation on the boundary wall and the boundary layer can be composed of the following criteria:

1. The criterion of similarity of radiation:

$$
K_{\text {rad }}=\frac{k \cdot d_{c}}{\frac{1}{\delta_{w}}-\frac{1}{2}}\left[1+\frac{T_{w}}{T}+\left(\frac{T_{w}}{T}\right)^{2}+\ldots+\left(\frac{T_{w}}{T}\right)^{n}\right]
$$

$k$-absorption coefficient; $\mathrm{d}_{\mathrm{c}}-$ combustion chamber diameter, $\mathrm{m}$

Based on the analysis in (3) the quantities and heat transfer characteristics with regard to small combustors radiation similarity criterion may be converted to the form

$$
K_{\text {rad }}=f(k \cdot d, \alpha)=f\left(\tau_{\mathrm{o}}, \alpha\right),
$$

$\alpha$ - excess air ratio; $\tau_{\mathrm{o}}=k \cdot d-$ optical thickness, $\mathrm{m}$.

2. Criterion turbulent and molecular transport:

$$
K_{\mathrm{T}-\mathrm{M}}=\frac{D_{t u r b} \cdot \rho\left(I_{t h}-I_{w}\right)}{\lambda_{\text {mol }}\left(T_{t h}-T_{w}\right)},
$$

that under these conditions can be expressed as

$$
K_{\mathrm{T}-\mathrm{M}}=f_{1}\left(\operatorname{Re}_{\mathrm{H}} ; \frac{d_{b}}{d_{c}} ; \frac{l}{d_{c}}\right),
$$

$\mathrm{Re}_{\mathrm{H}}-$ Reynolds number; $l$ - length of the chamber, $\mathrm{m}$.

3. The criterion of radiation and molecular transport:

$$
K_{\mathrm{R}-\mathrm{M}}=\frac{\sigma_{\mathrm{o}} n^{2} T^{3}}{k \lambda_{\text {mol }}} .
$$

In these conditions, $K_{\mathrm{R}-\mathrm{M}}$ has no effect on the heat transfer in the combustion chamber. For homogeneous combustion reactions influence reaction-kinetic factors on the heat transfer can be neglected [5] or to consider them identical for these combustion chambers with similar conditions of entry of reacting masses.

Thus, the similarity of the equation to determine heat transfer in this case can be represented as follows:

$$
K_{\mathrm{T}}=f\left(\operatorname{Re}_{\mathrm{H}} ; \tau_{o} ; \alpha ; \frac{d_{b}}{d_{c}} ; \frac{l}{d_{c}}\right),
$$

The functional dependence of the integral criterion of the transfer of numbersarguments can most reliably be determined on the basis of an experimental study of complex heat exchange on models and real plants.

Experimental study of complex radiation and convective heat transfer was carried out in the combustion chambers, executed in real (Fig. 1). Installation consists of water-cooled section combustion chambers 1 , burners on liquid and gaseous fuels for the different conditions of entry and combustion of the fuel-air mixture 2, devices for feeding and adjusting the air flow, fuel and cooling water, as well as instrumentation to determine pressure, flow rate, temperature and composition of fuel and combustion products.

The combustion chamber is a vertical cylindrical heat exchangers diameter $\mathrm{dc}-0.18$; $0.24 ; 0.3 ; 0.36 \mathrm{~m}$, collected from sections of different lengths in order to obtain middle zone 
of heat fluxes on surfaces corresponding to the relative length of the section $\frac{l}{d_{c}}=\frac{1}{3}$ calculated by determining the temperature and coolant flow at points "B".

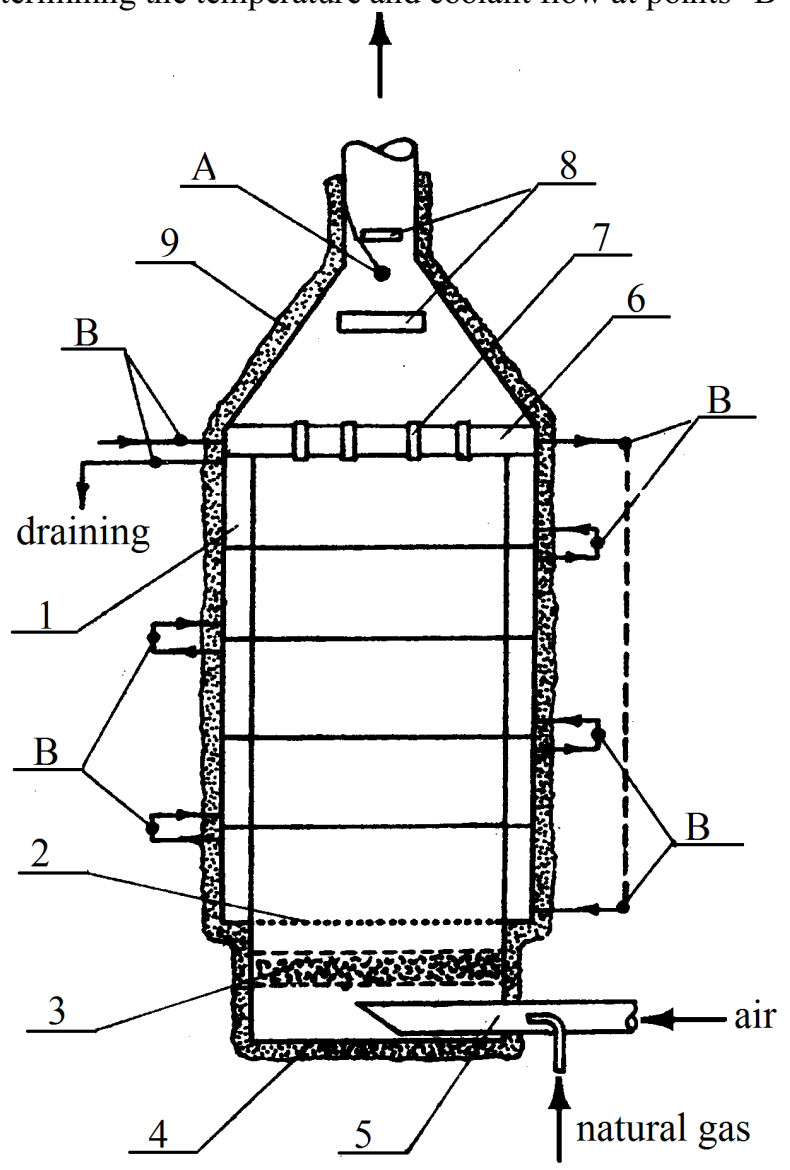

Fig. 1. Experimental stand.

To investigate the effect of heat on the length of the combustion chamber at constant thermal stresses furnace volume $q_{v}$ experimentally determined integrated heat transfer in cells of $\frac{l}{d_{c}}=1 ; 1 \frac{1}{3} ; 2 ; 2 \frac{1}{3}$ in length.

In the upper part of a hollow drive water cooled combustion chamber outlet calorimeter installed fire tubes 7 distributed on the surface so that the ratio of the cross sectional area of the combustion chamber and the outlet section area kept constant flue tubes for cameras of different diameters.

Flue gas temperature at the outlet of the cylindrical part of the definition-fissile as measured gas temperature at the point "A" located between the screens 8 and the largest heat absorption of the calorimeter output.

In one series of experiments the combustion chamber equipped with a burner full air pre-mixing with natural gas. The burner is composed of body 4, a mixer 5, a layer of ceramic chips 3 , for leveling the velocity field of air-fuel mixture and heating this mixture, 
nichrome mesh 2, which was carried out with kinetic combustion gas excess air factor $\alpha=$ $1.05-2.0$.

This equation (8) allows on the basis of generalization of experimental data to analyze the impact of regime and geometrical factors, as well as the properties of the absorbing medium in the heat in the cold combustion chamber under identical input conditions. A study in this publication and in [6] shows that the absorption coefficient $\mathrm{k}$ of the combustion products is calculated on the Rosseland diffusion approximation is inversely proportional to the magnitude of the coefficient of excess air. In this case, radiation similarity criterion may be represented as follows:

$$
K_{\text {rad }}=\frac{k \cdot d_{c}}{\alpha}
$$

Absorption coefficient of the combustion products at $\alpha=1.0$ is for natural gas $\mathrm{k}_{\mathrm{o}}=3.34$ $\mathrm{m}^{-1}$ and for oil $\mathrm{k}_{\mathrm{o}}=3.92 \mathrm{~m}^{-1}$.

Excess air ratio has different effects on the individual components of the complex heat flow [7].

With an increase in the excess air ratio is intensification of convective heat transfer due to the increase of Re criterion [8].

At the same time decreases the theoretical combustion temperature of the fuel and the partial pressure of triatomic gases, i.e., excess air ratio affects the magnitude of the thickness of the optical medium.

The introduction of excess air factor as an argument doesn't preclude consideration of Re criterion despite having a dependence of the criterion of $\alpha$. This is because at constant values $\alpha$ and $\frac{l}{d_{c}}$ determines the change in Re emitting medium flow mode, i.e. determines the intensity of convective heat transfer. Simultaneously, the criterion is a function of the thermal stress of the combustion chamber volume.

Excess air ratio at constant values of Re and $\frac{l}{d_{c}}$, but for different specific energy in the volume of the chamber represents the change in the radiation component of heat transfer in a complex.

The experimental data were processed by the method allows to determine the dependence of the radiation and convective components of the air-fuel ratio.

As a result of analysis of experimental data, the following dependence, reflecting the impact of the air excess factor $\alpha$ on heat transfer in the combustion chamber

$$
\begin{gathered}
K_{\mathrm{T}}=A\left(\frac{k \cdot d_{c}}{\alpha}\right)^{0,47} \text { at } \mathrm{Re}=\text { const } ; \frac{l}{d_{c}}=\text { const } \\
K_{\mathrm{T}}=B \operatorname{Re}_{\mathrm{H}}^{-0.33} \text { at } \alpha=\text { const } ; \frac{l}{d_{c}}=\text { const }
\end{gathered}
$$

As determined by gas velocity takes speed, per unit surface, which determines the size of the diameter of the receiving chamber, for determining the temperature - the theoretical combustion temperature.

Fig. 2 shows the effect of the coefficient $\alpha$ on radiation component at a constant value of the convective heat transfer. Equation (10) describes the dependence of the convective component of heat transfer $\operatorname{Re}_{\mathrm{H}}=f\left(q_{v}\right)$ from a small change in the intensity of radiation heat transfer. 


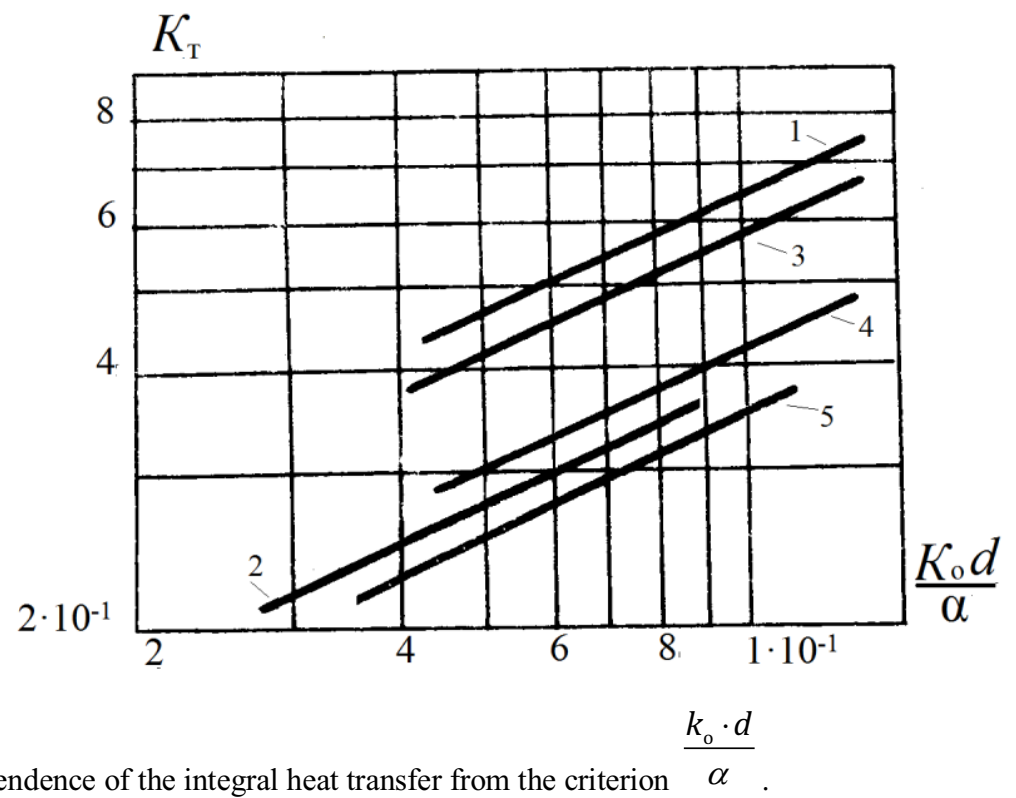
1) $\left.\frac{l}{d_{c}}=7 / 3, \mathrm{Re}_{\mathrm{H}}=67.1-80.1 ; 2\right) \frac{l}{d_{c}}=7 / 3, \mathrm{Re}_{\mathrm{H}}=299.1-315.2$;
3) $\frac{l}{d_{c}}=7 / 3, \mathrm{Re}_{\mathrm{H}}=121.1-137.1$; 4) $\frac{l}{d_{c}}=7 / 3, \mathrm{Re}_{\mathrm{H}}=120.6-144.4$;
5) $\frac{l}{d_{c}}=4 / 3, \mathrm{Re}_{\mathrm{H}}=189.9-211.3$.

Generalization of exponential function experimental data results helped brush up on private transport depending on the number of integrated $\mathrm{K}_{\mathrm{T}}$ from the criterion of numbers the arguments included in the similarity of the equation (3).

Processing results of experiments in combustors diameter $\mathrm{d}=0.18-0,36 \mathrm{~m}$, equipped with burners and incomplete full premixing of fuel and air, when distributed over the cross section entering fuel-air mixture generalized:

$$
K_{\mathrm{T}}=\frac{1}{1+0.1016 \cdot \mathrm{Re}_{\mathrm{H}}^{0.55} \cdot\left(\frac{k_{\mathrm{o}} \cdot d}{\alpha}\right)^{-0.86} \cdot\left(\frac{l}{d_{c}}\right)^{0.75}},
$$

In experiments on fuel combustion load is varied in the range of $1.5 \cdot 10^{-4}-17.5 \cdot 10^{-4}$ $\mathrm{kg} / \mathrm{s}$, the excess air factor $\alpha=1.05-2.0$, temperature at the exit from a fire chamber $\mathrm{T}_{2}=$ $1011-1752 \mathrm{~K}$, conditional Reynolds number $\mathrm{Re}_{\mathrm{H}}=66-359$, the relative length of the chamber $\frac{l}{d_{c}}=1-2 \frac{1}{3}$, radiation similarity criterion $\frac{k_{o} \cdot d}{\alpha}=0.301-1.145$, the number of heat transfer integral $K_{\mathrm{T}}=0.15-0.67$, specific energy in the chamber $\mathrm{q}_{y}=0.76-1.73$ $\mathrm{MW} / \mathrm{m}^{3}$. 
The resulting dependency generalizes the criterion (11) allows us to estimate the contribution of radiative and convective heat transfer component in the complex for lowpower boilers and combustion chambers to identify qualitative and quantitative dependence of the integrated radiative-convective heat transfer from the basic geometrical, physical and regime factors working combustion chambers of small volume.

Evaluation of the reliability of the experimental data showed that the value of the marginal mean square error in the determination of the integral heat transfer $K_{\mathrm{T}}$ will be $3.24 \%$. Correlation generalizing conformity assessment according to (11) was produced in the number of integrated $K_{\mathrm{T}}$ heat transfer and temperature of the gases at the outlet of the furnace $\mathrm{T}_{2}$. For all experiments, the deviation of the calculated data from the results of the experiment with a probability of $95 \%$ is within the confidence interval of $\pm 9.52 \%$.

\section{References}

1. R .Goulard, M. Goulard, Proceding of the Heat Transfer and Fluid Mechanics Institute Stanford University Press. Pelo Alto., Calit. (1959)

2. P.A. Khavanov, Sources of heat autonomous heating systems: monography (MGSU, Moscow, 2014)

3. H.M. Abu-Romia, C.L. Tien, Trans, of the ASME, ser. C, Dournel of Heat Transfer 4, (1967).

4. S.N. Shorin, V.I. Sukhov, Influence the torches on the characteristic of heat exchange in gas combustion chambers. Theory and practice of combustion of gas. Release 3 (Nedra, 1967)

5. O.N. Bruykhanov, S.N. Shevchenko, Heat and Mass Transfer. Textbook. (Infra-M, 2014)

6. N.M. Konovalova, S.N. Shorin, R.M. Shurin. Chemical engineering 1, (1974).

7. G. N. Delyagin, V. I. Lebedev, B.A. Permyakov, P. A. Havanov, Heat-generating installation. Studies for higher education institutions. 2nd ed. (Open company "Bastet's" IDES", Moscow, 2010)

8. V.V. Lemanov, V.I. Terekhov, THT 3, (2016) 